\section{BARRETT ESOPHAGUS}

\section{PEPTIDE SEQUENGE}

DETECTS DYSPLASIA

A peptide sequence that binds specifically to dysplasia in Barrett esophagus, and can be fluorescence labeled, has been identified by Thomas Wang and colleagues. This peptide sequence (SNFYMPL) could be useful for the early detection of cancer in Barrett esophagus.

Molecular imaging is becoming increasingly important as a diagnostic tool in clinical practice. Peptides have great potential for use as molecular probes because they are small and can be fluorescence labeled. They are also compatible with use in the digestive tract. Wang and colleagues have previously identified a fluorescencelabeled peptide sequence that was able to distinguish between dysplasia and normal colonic mucosa in vivo using a confocal microendoscope.

The same group decided to investigate whether a similar strategy might work for detecting dysplasia in Barrett esophagus. "The incidence of esophageal adenocarcinoma is increasing at an unprecedented rate, and current surveillance methods with random biopsy on white light endoscopy are inadequate," says Wang, corresponding author. "Targeted imaging could guide biopsies and improve our ability to catch early lesions."

The researchers used phage display to select short-chain peptides that bound with high affinity to overexpressed molecular targets on the surface of premalignant esophageal cells. Using this approach, the peptide sequence SNFYMPL was identified as binding specifically to dysplasia in Barrett esophagus. The selective binding of SNFYMLP was confirmed by enzymelinked immunosorbent assay, flow cytometry and confocal microscopy.

"We plan to take this work forward by validating the use of this peptide in a clinical study for the early detection of cancer in Barrett esophagus," concludes Wang.

Isobel Franks

Original article Li, M. et al. Affinity peptide for targeted detection of dysplasia in Barrett's esophagus. Gastroenterology 139, 1472-1480 (2010) 